\title{
Análisis dinámico en el remo de banco fijo: la trainera Dynamic analysis on the fixed seat rowing: trainera
}

\author{
Héctor Lorenzo Buceta*, Sergio Pérez Treus*, José Luís García Soidán*, Víctor Arufe Giraldez**, Xavier Alfonso \\ Cornes***,AlexandreAlfonso Cornes*** \\ *Universidad de Vigo. **Universidad de La Coruña. ***UMANA, Centro sanitario de investigación biomecánica
}

Resumen: El remo de banco fijo es una modalidad deportiva no olímpica que tiene su mayor potencial en las regiones del norte de España. La acelerometría se basa en la capacidad de cuantificar de manera objetiva variables técnicas, factores mecánicos y biomecánicos. El objeto de estudio de esta investigación es el de analizar las componentes dinámicas de la fuerza, potencia, aceleración que actúan sobre el conjunto de la embarcación y remeros en la prueba de trainera, para así poder determinar las exigencias de esta competición. Tras el análisis de las 633 paladas que se dieron en una prueba de 5556 metros de distancia se encontró un promedio de fuerza máxima de palada de $4596.85 \pm 552.85 N$. Se encontraron diferencias significativas ( $p<.001)$ entre el número de paladas de cada largo, siendo el segundo en donde se realizan menos paladas, así como en donde menos se aplica la fuerza en la palada. En la fuerza máxima de palada se encontraron diferencias significativas ( $p<.001)$ con respecto al largo, siendo el último largo en donde se aplica más fuerza máxima de palada. El segundo largo, aun teniendo una aplicación de fuerza menor, se encontraron mayores niveles de avance por palada, en relación con los otros tres largos. Esta investigación es un punto de partida para la implementación de la acelerometría en el remo de banco fijo, dado que sus valores prestan importancia en los aspectos técnicos y condicionales, pudiendo obtener valores para determinar los niveles de fatiga en cada uno de los largos de la competición.

Palabras Clave: Remo banco fijo, acelerometría, biomecánica

Abstract: The fixed seat rowing is not a sport that has its largest Olympic potential in the northern regions of Spain. The accelerometer is based on the ability to objectively quantify technical variables, mechanical and biomechanical factors. The purpose of this research study is to analyze the dynamic components of strength, power, acceleration acting on the set of the boat and rowers in testing trainera, in order to determine the requirements of the competition. After analysis of the 633 strokes that occurred in a test of 5556 meters away found an average maximum force shovelful of $4596.85 \pm 552.85 \mathrm{~N}$. There were significant differences $(p<.001)$ between the number of strokes of each length, the second where they make fewer strokes and where less force is applied to the stroke. The maximum force of stroke were significant differences $(p<.001)$ compared to long, the last long where more force is applied maximum stroke. The second length, while having a lower force application, they found high levels of feed per stroke in relation to the other three times. This research is a starting point for the implementation of the accelerometer in the fixed seat rowing, since their values give importance on the technical aspects and conditional, being able to get values to determine fatigue levels in each of the lengths of the competition. Key words: fixed seat rowing, accelerometry, biomechanics

\section{Introducción}

El remo de banco fijo es una modalidad deportiva no olímpica que tiene su mayor potencial en las regiones del norte de España. En la actualidad, el remo de banco fijo, aun no siendo disciplina olímpica, se caracteriza por primar el factor económico con gran perseverancia.

El remo de banco fijo del cantábrico tiene tres modalidades: (1) batel, (2) trainerilla y (3) trainera. Esta última, formada por 13 remeros y 1 patrón, y la prueba se basa en recorrer una distancia de 5556 metros distribuida en cuatro largos (FER, 2011).

Todo deportista busca una mejora continua del rendimiento y de los resultados de competición, esta mejora se alcanza a través de una preparación física, psíquica y técnico-táctica (Weineck, 2005). El control del rendimiento, así como la mejora de los aspectos técnicos se pueden complementar con la aplicación de la ingeniería al ámbito del deporte, así, existen numerosos estudios en los que constatan la cuantificación objetiva de cada uno de los parámetros que influyen de manera significativa en el rendimiento de los deportistas (Vanhelst, Theunynck, Gottrand \& Béghin, 2010; Cook, Alberts \& Lambert, 2012).

En la literatura científica se encuentra que la acelerometría es aplicada en numerosos deportes (Pérez, et al, 2012; Boyd, Ball \& Aughey, 2013; Callaway, Cobb \& Jones, 2009), así como en la actividad física para la salud y en la prevención del sedentarismo y los riesgos cardiovasculares (Tanaka Hikihara, Ohkawara \& Tanaka, 2012; Tan, Batterham \& Tapsell, 2011; Pruitt, et al, 2008).

La acelerometría se basa en la capacidad de cuantificar de manera objetiva variables técnicas, factores mecánicos y biomecánicos, asícomo

Fecha recepción: 28-07-13- Fecha envío revisores: 28-07-13- Fecha de aceptación: 08-09-13 Sergio Pérez Treus

Carretera General, Escarabotiñ, 44

15992 Boiro - A Coruña

sergiopereztreus@gmail.com información acerca de la intensidad, frecuencia y duración de la actividad desarrollada, y así, mediante esta cuantificación poder realizar una mejor distribución de los remeros para la mejora del desplazamiento y la técnica en la embarcación (Chen \& Basset, 2005).

Otros autores (Sanderson \& Martindale, 1986; Affeld, Schichl \& Ziemann, 1993) destacan que la eficiencia externa o propulsora de la palada en conexión con la hidrodinámica del casco de la embarcación y el remo están estimadas en un rango de 60 a 80\%, pudiéndose estimar esta mediante la aplicación de la acelerometría en la embarcación.

Kleshnev (1999) en su estudio descubrió que los dos factores más influyentes en la eficiencia de la palada en banco móvil fueron la frecuencia de palada y la duración de la fase de pasada del remo durante la frecuencia de estas paladas.

Dentro del remo de banco móvil se encuentra estudios de diversa índole en la aplicación biomecánica y de cuantificación de las fuerzas en la embarcación (Baudouin \& Hawkins, 2004; Hill \& Fahrig, 2009; Doyle, Lyttle \& Elliott, 2010). Los remeros necesitan un constante feedback sobre su cinemática de palada para así poder corregir los errores técnicos (Anderson, Harrison \& Lyons, 2005). Las mediciones mediante acelerómetros triaxiales ayudan a mejorar los aspectos técnicos en el remo de banco fijo.

Smith y Loschner (2002) demostraron que un buen feedback biomecánico en el remo de banco móvil sobre la aplicación de las fuerzas en la palada tenía un efecto significativo sobre la velocidad de la embarcación, siendo esta mayor al tener un feedback en la aplicación de fuerza.

Durante la fase de pasada del remo sobre la lámina de agua el remero produce una acción de fuerza en el barco como resultado de la interacción de sus fuerzas dadas sobre el tolete, las fuerzas en la bancada de los pies y el asiento y las fuerzas de las fuerzas exteriores de la pala (Mattes \& Schaffert, 2010). Todo el conjunto de esas fuerzas de cada uno de los remeros de las embarcaciones (batel, trainerilla y trainera), hace acelerar el sistema (barco y remeros).

Colloud, Bahuaud, Doriot, Champeli y Chéze (2006) encontraron valores en test de remoergómetro en banco fijo de $1134 \pm 108 \mathrm{~N}$ de 
fuerza y 2491 $\pm 299 \mathrm{~W}$ de potencia, ambos valores analizados en la dirección antero-posterior. Kleshnev (2000) destaca que el total de potencia aplicada en la embarcación de remo de banco móvil mediante los pies y los brazos está distribuida en un $47.2 \pm 4.1 \%$ y $52.8 \pm 4.1 \%$, respectivamente. En el análisis de las fuerzas en el remo se pueden medir hasta tres componentes (hacia delante, lateral y vertical) (Smith \& Loschner, 2000).

Pérez et al. (2012) realizaron un estudio similar en el piragüismo de aguas tranquilas en donde analizaban la prueba de 200 metros.

Estos parámetros de estudio son de gran importancia a la hora de la determinación de la producción de energía desarrollada por todo el conjunto de la embarcación durante toda la prueba, así como la determinación de la fatiga durante el transcurso de la prueba de trainera.

El objetivo de este estudio fue el de analizar las componentes dinámicas de la fuerza, potencia, aceleración que actúan sobre el conjunto de la embarcación y remeros en la prueba de trainera, para así poder determinar las exigencias de esta competición.

\section{Material y método}

Se diseñó un estudio descriptivo transversal, para intentar medir las fuerzas máximas que intervienen en el desplazamiento de una trainera de competición.

\section{Participantes}

Este estudio se llevó a cabo con 13 remeros y 1 patrón en la modalidad de trainera dela disciplina del remo de banco fijo del cantábrico. El conjunto de trainera y remeros tenían un peso de 1306kg. Los remeros tenían una edad promedio de $23.02 \pm 6.18$ años, una talla de $178.5 \pm 7.82 \mathrm{~cm}$, un peso de $75.98 \pm 8.86 \mathrm{~kg}$ y una envergadura de $181.44 \pm 10.07$.

\section{Instrumento}

Para la adquisición y tratamiento de datos se utilizó un sistema de acelerometría triaxial Serie UP/CK/V1 (Umana Innova) y un software de análisis de datos UP/CK/V1 - AIM RS2 (Umana Innova). La prueba se realizó en una Trainera modelo «Pedro Cuesta» fabricada en el año 1993. Su peso total fue de $206 \mathrm{~kg}$.

\section{Procedimiento}

El test se realizó en una lámina de agua estable sin ningún viento, sobre una distancia de 5556 metros, distribuidos en cuatro largos de 1389 metros. Antes de la realización de la prueba, se comprobó el correcto anclaje del sistema de acelerometría con unas bandas dual lock a la base de la trainera (figura 1), para que no se produjese ningún movimiento durante el test. El sistema de captura de datos está formado por tres acelerómetros, para la valoración de los tres ejes, un GPS para contabilizar la velocidad y un giroscopio para la medición de los ángulos.

Para el análisis de los datos se dividió la prueba en cuatro largos y tres ciabogas. Las ciabogas se consideraron desde que el proel introducía

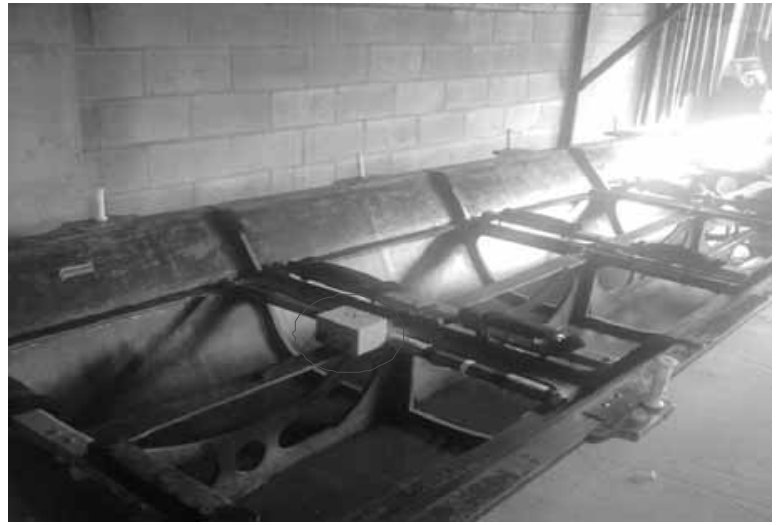

Figura 1. Colocación del acelerómetro triaxial Serie UP/CK/V1 (Umana Innova) en la trai nera la pica en la proa hasta que la trainera salía recta en el siguiente largo. La prueba comenzó con un calentamiento de 20 minutos de duración, dividida en una fase de calentamiento general a través de ejercicios de movilización articular global, y una parte de calentamiento específico, destinada al acondicionamiento del organismo (frecuencia cardíaca, frecuencia respiratoria, etc.), el sistema articular (cápsulas, ligamentos), y los músculos implicados en la realización de la actividad deportiva del remero (Pérez, et al., 2012).

Antes de comenzar el test, se procedió a encender y comprobar la detección de la señal GPS de la base de captura de datos UP/CK/V1 (Umana Innova). La prueba se inició con la embarcación totalmente parada y con la proa de la misma en la línea que marcaba el inicio.

La orden de salida se emitió de forma sonora y en todo momento la prueba fue seguida por una videocámara $\mathrm{HD}$, con una frecuencia de captura que coincidía con el GPS, siguiendo un modelo ya presentado en otros estudios (Janssen \& Sachlikidis, 2010).

\section{Eficiencia de la embarcación}

Para ello se contó con la ecuación citada por Kleshnev (1999) en donde estableció la relación existente entre la frecuencia de paleo y la duración de la palada en la fase de tracción.

\section{Eficiencia $=\frac{\text { Duración palada }}{\text { Frecuencia palada }}($ Kleshnev, 1999)}

Este autor destaca que ante una disminución de esta relación se produce una ganancia en la eficiencia de la embarcación.

\section{Análisis de los datos}

Para el análisis de los datos se utilizó el software UP/CK/V1 -AIM RS2 (Umana Innova), con el que se analizaron las variables que se presentan en la siguiente tabla (tabla 1).

\begin{tabular}{|c|c|c|c|}
\hline $\mathbf{N}^{\circ}$ & Parámetro & Unidad Medida & Incertidumbre \\
\hline 1 & Ampli tud pal ada & $\mathrm{m}$ & \\
\hline 2 & Duración palada & $\mathrm{s}$ & \\
\hline 3 & Aceleración & G & $+.01 G$ \\
\hline 4 & Velocidad & $\mathrm{m} / \mathrm{s}$ & $\pm .07 \frac{\mathrm{m}}{-}$ \\
\hline 5 & Fuerza máxima palada & $\mathrm{N}$ & $+.05 \stackrel{\varsigma}{N}$ \\
\hline 6 & Potencia máxi ma palada & w & $\underline{ \pm} .0001 \mathrm{~W}$ \\
\hline 7 & Potencia relat iva palada & $\mathrm{W} / \mathrm{kg}$ & \\
\hline 8 & Frecuencia de paleo & $\mathrm{ppm}$ & \\
\hline
\end{tabular}

\begin{tabular}{lccccc}
\multicolumn{7}{c}{ Tabla 2. 2Análisis de las variables de estudio en la prueba de trainera } \\
\hline Variables & N & Mínimo & Máximo & Media & DT \\
\hline Amplitud $(\mathrm{m})$ & 633 & 2.14 & 10.13 & 8.79 & 1.14 \\
Tiempo $(\mathrm{s})$ & 633 & 1.48 & 2.32 & 2.07 & .10 \\
Aceleración $\left(\mathrm{m} / \mathrm{s}^{2}\right)$ & 633 & .59 & 4.41 & 3.51 & .42 \\
Velocidad $(\mathrm{m} / \mathrm{s})$ & 633 & 1.58 & 4.83 & 4.42 & .47 \\
Fuerza (N) & 633 & 767.93 & 5759.46 & 4596.85 & 552.85 \\
Potencia (W) & 633 & 1388.67 & 27645.41 & 20544.53 & 3694.16 \\
Potencia Rel ativa (W/kg) & 633 & 1.06 & 21.17 & 15.73 & 2.82 \\
Kg fuerza aplicada palada (9.81·N) & 633 & 78.36 & 587.70 & 469.06 & 56.41 \\
Frecuencia de palada (ppm) & 633 & 26.00 & 41.00 & 28.95 & 1.66 \\
\hline
\end{tabular}

Para el análisis de los datos recogidos mediante la base de captura UP/CK/V1 (Umana Innova) se siguió el modelo presentado por Pérez et al. (2012), en donde se extrapolan los datos procedentes de la aceleración en el eje X y la velocidad(GPS), para así realizar en análisis de las variables de la tabla 1.

Tras la recogida de los datos y el posterior análisis en el software UP/CK/V1-AIM RS2 (Umana Innova), se utilizó el paquete informático SPSS para Windows versión 20.0 (SPSS Inc. Chicago, IL, USA) utilizando medidas de tendencia central (mínimo, máximo, media y desviación típica). Se aplicóla prueba de homocedestacidad (prueba de Levene) y tras esta se realizó un ANOVA teniendo como factor los largos y ciabogas realizadas, utilizando el «post hoc» de Bonferroni, asumiendo igualdad de varianzas. Asícomo también lapruebade correlación bivariada de Pearson. Se estableció el nivel crítico de significación en $p<.05$. 


\begin{tabular}{|c|c|c|c|c|c|}
\hline Variables & $\mathbf{N}$ & Mínimo & Máximo & Media & DT \\
\hline Amplitud (m) & 152 & 2.14 & 9.82 & 8.81 & .88 \\
\hline Tiempo (s) & 152 & 1.64 & 2.30 & 2.06 & .12 \\
\hline Aceleración $\left(\mathrm{m} / \mathrm{s}^{2}\right)$ & 152 & .59 & 4.21 & 3.51 & \\
\hline Velocidad $(\mathrm{m} / \mathrm{s})$ & 152 & 1.81 & 4.82 & 4.46 & 0.27 \\
\hline Largo 1 Fuerza (N) & 152 & 767.93 & 5503.48 & 4589.04 & 450.50 \\
\hline Potencia (W) & 152 & 1388.67 & 26294.42 & 20571.10 & 2485.49 \\
\hline Potencia Relativa (W/kg) & 152 & 1.06 & 20.13 & 15.75 & 1.90 \\
\hline Kg fuerza aplicada palada $(9.81 \cdot \mathrm{N})$ & 152 & 78.36 & 561.58 & 468.26 & 45.96 \\
\hline Frecuencia de palada (ppm) & 152 & 26.00 & 37.00 & 29.19 & 2.03 \\
\hline Amplitud (m) & 149 & 4.59 & 10.13 & 9.36 & .76 \\
\hline Tiempo (s) & 149 & 1.80 & 2.32 & 2.15 & .08 \\
\hline Aceleración $\left(\mathrm{m} / \mathrm{s}^{2}\right)$ & 149 & 2.45 & 4.02 & 3.47 & .24 \\
\hline Velocidad $(\mathrm{m} / \mathrm{s})$ & 149 & 2.73 & 4.78 & 4.54 & .24 \\
\hline Largo 2 Fuerza (N) & 149 & 3199.70 & 5247.51 & 4532.83 & 322.93 \\
\hline Potencia (W) & 149 & 8719.18 & 24269.72 & 20661.67 & 2096.25 \\
\hline Potencia Relativa (W/kg) & 149 & 6.68 & 18.58 & 15.82 & 1.60 \\
\hline Kg fuerza aplicada palada $(9.81 \cdot \mathrm{N})$ & 149 & 326.50 & 535.46 & 462.53 & 32.95 \\
\hline Frecuencia de palada (ppm) & 149 & 26.00 & 33.00 & 27.84 & 1.06 \\
\hline Ampl itud (m) & 157 & 4.38 & 9.57 & 8.56 & .70 \\
\hline Tiempo (s) & 157 & 1.78 & 2.26 & 2.09 & .07 \\
\hline Aceleración $\left(\mathrm{m} / \mathrm{s}^{2}\right)$ & 157 & 2.25 & 3.92 & 3.48 & .27 \\
\hline Velocidad $(\mathrm{m} / \mathrm{s})$ & 157 & 2.61 & 4.53 & 4.29 & .25 \\
\hline Largo 3 Fuerza (N) & 157 & 2943.72 & 5119.52 & 4552.94 & 357.68 \\
\hline Potencia (W) & 157 & 7670.04 & 22895.63 & 19631.05 & 2298.47 \\
\hline Potencia Relativa (W/kg) & 157 & 5.87 & 17.53 & 15.03 & 1.75 \\
\hline $\mathrm{Kg}$ fuerza aplicada palada $(9.81 \cdot \mathrm{N})$ & 157 & 300.38 & 522.40 & 464.58 & 36.49 \\
\hline Frecuencia de palada (ppm) & 157 & 27.00 & 34.00 & 28.91 & 1.20 \\
\hline Amplitud (m) & 157 & 3.87 & 10.09 & 8.95 & .76 \\
\hline Tiempo (s) & 157 & 1.48 & 2.23 & 2.02 & .08 \\
\hline Acel eración (m/s²) & 157 & 2.35 & 4.41 & 3.79 & .29 \\
\hline Velocidad (m/s) & 157 & 2.60 & 4.83 & 4.63 & .26 \\
\hline Largo 4 Fuerza(N) & 157 & 3071.71 & 5759.46 & 4950.77 & 388.86 \\
\hline Potencia (W) & 157 & 7977.92 & 27645.41 & 23026.69 & 2602.71 \\
\hline Potencia Relativa (W/kg) & 157 & 6.11 & 21.17 & 17.63 & 1.99 \\
\hline Kg fuerza aplicada palada ( 9 . & 157 & 313.44 & 587.70 & 505.18 & 39.68 \\
\hline Frecuencia de palada (ppm) & 157 & $\begin{array}{l}27.00 \\
\end{array}$ & 41.00 & $\begin{array}{l}29.77 \\
\end{array}$ & $\begin{array}{l}1.640 \\
1.64\end{array}$ \\
\hline
\end{tabular}

\section{Resultados}

Tras el análisis de las 633 paladas pertenecientes a la prueba de trainera sobre la distancia de 5556 metros, se pueden observar en la siguiente tabla (tabla 2) los valores obtenidos.

Teniendo en cuenta el largo realizado (tabla 3), se observaron diferencias significativas $(p<.001)$ entre los cuatro largos y el número de paladas aplicadas. El tercer y cuarto largo fueron los largos con mayor número de paladas (157), seguido por el primer (152), donde el largo con menos paladas fue el segundo (149).

Fuerza aplicada

En lo referido a la fuerza aplicada (Figura 2), en el total de la prueba se encontró un promedio de $4596.85 \pm 552.5 N$. Se encontraron diferencias estadísticamente significativas ( $p<.001)$ entre la fuerza aplicada en el cuarto largo y el resto de los largos, teniendo en este último largo una fuerza máxima promedio aplicada de 4950.77£388.86, siendo este largo en donde más se aplicaba fuerza.

Dentro de las tres ciabogas realizadas no se encontraron diferencias estadísticamente significativas ( $p>$.05) entre las variables de estudio, pudiendo observarse que la primera ciaboga es donde se genera mayor fuerza aplicada, siendo la tercera, y última ciaboga, en donde se produce una fuerza neta aplicada menor.

\section{Potencia aplicada}

La potencia promedio de toda la prueba estuvo situada en 20544.53 $\pm 3694.16 \mathrm{~W}$, siendo el segundo largo en donde se aplica mayor

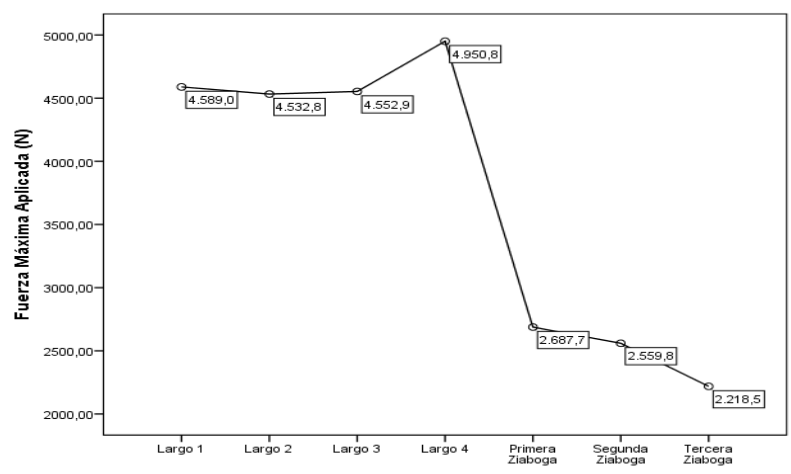

Figura 2. Dis tri bución de la fuerza máxima promedio potencia promedio.

En lo que respecta a la potencia aplicada en el conjunto de toda la embarcación, se encontraron diferencias significativas $(p<.001)$ entre los cuatro largos, siendo el tercer largo en el que menor aplicación de potencia se encuentra.

Amplitud y duración de palada

Por otro lado, se encontraron diferencias significativas $(p<.001)$ entre la amplitud de la palada y los largos, siendo el segundo largo donde mayor amplitud se encuentra ( $9.36 \mathrm{~m})$, encontrando el tercer largo con la menor amplitud de la palada ( $8.56 \mathrm{~m})$.

En lo referido a la duración de la palada, se encontrar diferencias significativas $(p<.01)$ entre los diferentes largos, siendo el segundo largo en donde la palada es más lenta (2.15s) y el cuarto largo donde es más rápida (2.02s).

Siguiendo a Kleshnev (1999) se establecieron los factores que influencian en la eficiencia de la embarcación. Estos parámetros fueron la frecuencia del paleo y la duración de la palada, estando dichos parámetros correlacionados significativamente entre sí $(\mathrm{r}=-.63 ; p<.01)$. Se encontraron diferencias significativas $(p<.01)$ entre los cuatro largos, siendo el cuarto largo en donde se produce mayor eficiencia de la embarcación.

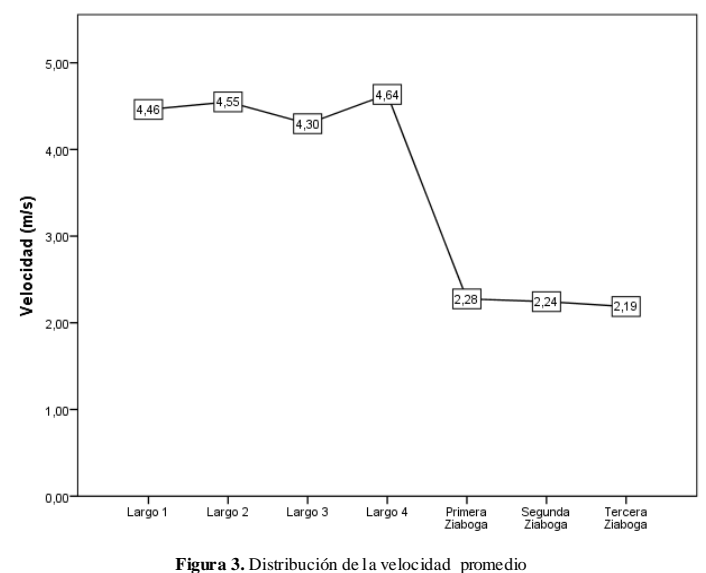

\section{Discusión y conclusiones}

El objetivo de esta investigación fue el de analizar las componentes dinámicas de la fuerza, potencia, aceleración que actúan sobre el conjunto de la embarcación y remeros en la prueba de trainera, para así poder determinar las exigencias de esta competición. De forma general podemos observar que se presta poca atención a la investigación mediante acelerometría en el deporte y en especial al remo (Pelham, Holt, Burke \& Carter, 1999; Mattes \& Schaffert, 2010; Kleshnev, 2000; Hill \& Fahrig, 2009, Bauduin \& Hawkins, 2004). En lo que respecta al banco fijo, en la literatura científica no encontramos ningún trabajo al respecto en la aplicación de la acelerometría para la valoración de las fuerzas aplicadas. Encontramos un artículo de Colloud et al. (2009), realizado en remoergómetro donde medían aspectos mecánicos con el asiento móvil y el asiento fijo, encontrando mayores niveles de fuerzas aplicadas, en el asiento fijo. En este estudio se encontraron valores promedios de $4596.85 \pm 552.85 \mathrm{~N}$ de fuerza máxima aplicada, durante el transcurso de una prueba de trainera.

Debemos señalar, que se encontraron diferencias estadísticamente significativas $(p<.001)$ en la producción de la fuerza entre un largo y otro, siendo el cuarto largo en donde se aplica mayor cantidad de fuerza y el segundo largo donde la aplicación de la fuerza fue menor (Figura 2).

Por otro lado, el segundo largo, aun teniendo una aplicación de fuerza menor, fue donde se encontraron los mayores niveles de avance por palada, en relación con los otros tres largos (Figura 4). En lo que respecta a la velocidad de la embarcación, se puede observar que el tercer largo tiene un promedio menor de velocidad $\left(4.30 \mathrm{~m} / \mathrm{s}^{2}\right)$ que los otros largos(Figura 3). 


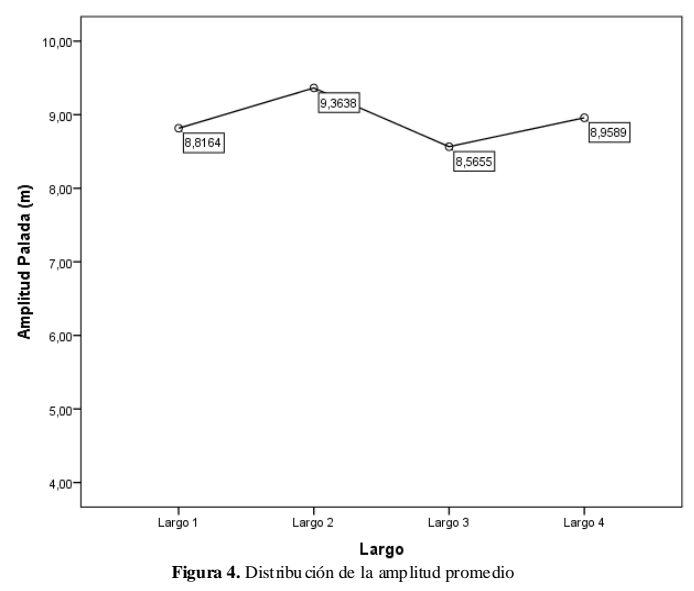

Las ciabogas son un lugar crucial a la hora del éxito de la competición, en esta muestra de estudio los sujetos realizaron 6 paladas durante la ciaboga, y se puede observar que no hay diferencias significativas en las tres ciabogas, aunque la tercera ciaboga es en donde menor fuerza máxima de palada se aplica, mientras que el cuarto largo es el más rápido (Figura 2).

Se pudo observar que en la tercera ciaboga fue donde se encontraron los valores más bajos de fuerza neta aplicada, encontrándose a continuación, en el último largo, los valores más altos de fuerza neta aplicada, esto constata que la última ciaboga los remeros la toman con un gasto energético menor que las ciabogas anteriores para afrontar el último y decisivo largo con menor fatiga.

Otro parámetro que da información acerca de la fatiga, es la relación entre la duración y la fuerza de la palada, teniendo en cuenta que en el remo estas dos variables van muy parejas, y es así, que se encontraron los valores promedios más bajos de la duración de la palada (2.15 .08s) en el segundo largo, donde además la fuerza máxima aplicada también es la más baja (4532.83 \pm 322.93$)$.

Siguiendo a Kleshnev (1999) y la relación que propone entre la frecuencia de la palada y la duración de la palada en esa fase de tracción para la eficiencia de la embarcación, se encontró en el cuarto largo la mejor eficiencia en la embarcación encontrando en dicho largo la mayor aplicación promedio de fuerza así como la palada más rápida.

En conclusión, esta investigación puede considerarse como un punto de partida para la implementación de la acelerometría en el remo de banco fijo, teniendo en cuenta que sus valores tienen importancia para los aspectos técnicos y condicionales. Con estos análisis se puede observar donde decrece la velocidad de la embarcación, donde se aplica menor fuerza y cuál es el largo más lento. La relación entre avance y fuerza aplicada, es un factor de suma importancia para poder observar la correcta aplicación de la fuerza durante el paleo.

Los trabajos basados en la acelerometría y la medición de la aceleración, fuerza aplicada son parámetros muy productibles a la hora de la valoración del rendimiento del conjunto de la embarcación, tanto técnico como orgánico, por eso sería una pieza imprescindible dentro del entrenamiento, en busca del mayor rendimiento de nuestros remeros.

Las futuras líneas de investigación están enfocadas a realizar un análisis pormenorizado a todas y cada unas de las modalidades del remo de banco fijo, para poder determinar los patrones de rendimiento biomecánico, fisiológico y técnico que esta tecnología nos puede dar Además, la valoración del rendimiento mediante acelerometría puede ayudar a la determinación de la palada más eficiente para cada una de las modalidades del remo, tanto de banco fijo como de banco móvil.

\section{Referencias}

Affeld, K., Schichl, K., \& Ziemann, A. (1993). Assessment of rowing efficiency. International journal of sports medicine, 14, S39 S41.

Anderson, R., Harrison, A., \& Lyons, G.M. (2005). Accelerometrybased feedback - Can it improve movement consistency and performance in rowing?. Sport Biomechanics, 4(2), 179-195.

Boudoin, A., \& Hawkins, D. (2004). Investigation of biomechanical factors affecting rowing performance. Journal of Biomechanics,

\section{7, 969-976.}

Boyd, L., Ball, K., \& Aughey, R. (2013). Quantifying External Load in Australian Football Matches and Training Using Accelerometers. International Journal of Sports Physiology and Performance, 8, 44-51.

Callaway, A., Cobb, J., \& Jones, I. (2009). A Comparison of Video and Accelerometer Based Approaches Applied to Performance Monitoring in Swimming. International Journal of Sports Science \& Coaching, 4(1), 139-153.

Chen, K.Y., \& Basett, D.R. (2005). The Technology of AccelerometryBased Activity Monitors: Current and Future. Medicine and Science in Sports \& Exercise, 34(11), 490-500.

Cook, I., Alberts, M., \& Lambert, S. (2012). Influence of Cut-points on Patterns of Accelerometry-Measurament Free-Living physical activity in rural and urban black south African women. Journal of physical activity and Health, 9, 300-310.

Colloud, F., Bahuaud, P., Doriot, N., Champeli, S., \& Chéze, N. (2006). Fixed Versus free-floating stretcher mechanism in rowing ergometers: Mechanical aspects. Journal of Sport Science, 24(5), 479-493.

Doyle, M.M., Lyttle, A., \& Elliott, B. (2010). Comparison of forcerelated performance indicators between heavyweight and lightweight rowers. Spots Biomechanics, 9(3), 178-192.

Federación Española de Remo (2011) Código de regatas de la Federación Española de Remo. Disponible en www.federemo.org

Hill, H., \& Fahrig, S. (2009). The impact of fluctuations in boat velocity during the rowing cycle on race time. Scandinavian Journal of Medicine and Science in Sports, 19, 585-594.

Janssen, I., \& Sachlikidis, A. (2010). Validity and reliability of intrastroke kayak velocity and acceleration using a GPS-based accelerometer. Sports Biomechanics, 9(1), 47-56.

Kleshnev, V. (1999). Propulsive efficience of rowing. Proc. XVII International Symposium on Biomechanics in Sports, Australia, June 30 - July 6.

Kleshnev, V. (2000). Power in rowing. Proc. XVIII Congress of ISBS, Hong Kong, Vol.II, 662-666.

Mattes, K., \& Schaffert, N. (2010). New measuring on water coaching device for rowing. Journal of Human Sport and Exercise, 5(2), 226-239.

Pelham, T.W., Holt, L.E., Burke, D.G., \& Carter, G.W. (1999). Accelerometry for paddling and rowing. XI International Symposium on Biomechanics in Sports, Amherst, Massachusetts - USA.

Pérez, S., García-Soidán, J.L., Arce, E., Portela, T., Alfonso, X., \& Alfonso, A. (2012). Acelerometría aplicada en el piragüismo de aguas tranquilas. IV Congreso Internacional de entrenadores de piragüismo de aguas tranquilas, Catoira. Pontevedra.

Pruitt, L., Glynn, N., King, A., Guralnik, J., Aiken, E., Miller, G., \& Haskell, W. (2008). Use of Accelerometry to Measure Physical Activity in Older Adults at Risk for Mobility Disability. Journal of Aging and Physical Activity, 16, 416-434.

Sanderson, B., \& Martindale, W. (1986). Towards optimizing rowing technique. Medicine and science in sports and exercise, 18, 454468.

Smith, R., \& Loschner, C. (2000). Net Power Production \& Performance at Different Stroke Rates \& Abilities During Sculling. XVIII International Symposium on Biomechanics in Sport, Hong Kong. China.

Tan, S., Batterham, M., \& Tapsell, L. (2011). Activity Counts From Accelerometers Do Not Add Value to Energy Expenditure Predictions in Sedentary Overweight Individuals During Weight Loss Interventions. Journal of Physical Activity and Health, 8, 675-681.

Tanaka, C., Hikihara, Y., Ohkawara, K., \& Tanaka, S. (2012). Locomotive and Non-Locomotive Activity as Determined by Triaxial Accelerometry and Physical Fitness in Japanese Preschool Children. Pediatric Exercise Science, 24, 420-434.

Vanhelst, J., Theunynck, D., Gottrand, F., \& Béghin, L. (2010). Reliability of the RT3 accelerometer for measurement of physical activity in adolescent. Journal of sport science, 28(4), 375-379.

Weineck, J. (2005). Entrenamiento Total. Barcelona: Paidotribo. 